\title{
Comparison of Event-related Changes in Oscillatory Activity During Different Cognitive Imaginary Movements Within Same Lower- Limb
}

\author{
Madiha Tariq, Pavel M Trivailo, Yutaka Shoji and Milan Simic
}

School of Engineering, RMIT University

264 Plenty Road, Bundoora, VIC 3083, Australia

s3519022@student.rmit.edu.au, pavel.trivailo@rmit.edu.au, s3278605@student.rmit.edu.au,milan.simic@rmit.edu.au

\begin{abstract}
The lower-limb representation area in the human sensorimotor cortex has all joints very closely located to each other. This makes the discrimination of cognitive states during different motor imagery tasks within the same limb, very challenging; particularly when using electroencephalography (EEG) signals, as they share close spatial representations. Following that more research is needed in this area, as successfully discriminating different imaginary movements within the same limb, in form of a single cognitive entity, could potentially increase the dimensionality of control signals in a braincomputer interface (BCI) system. This report presents our research outcomes in the discrimination of left foot-knee vs. right foot-knee movement imagery signals extracted from EEG. Each cognitive state task outcome was evaluated by the analysis of eventrelated desynchronization (ERD) and event-related synchronization (ERS). Results reflecting prominent ERD/ERS, to draw the difference between each cognitive task, are presented in the form of topographical scalp plots and average time course of percentage power ERD/ERS. Possibility of any contralateral dominance during each task was also investigated. We have compared the topographical distributions and based on the results we were able to distinguish between the activation of different cortical areas during foot and knee movement imagery tasks. Currently, there are no reports in the literature on discrimination of different tasks within the same lower-limb. Hence, an attempt towards getting a step closer to this has been done. Presented results could be the basis for control signals used in a cognitive infocommunication (CogInfoCom) system to restore locomotion function in a wearable lower-limb rehabilitation system, which can assist patients with spinal cord injury (SCI).
\end{abstract}

Keywords: Cognitive state; motor imagery; electroencephalography; brain-computer interface; event-related desynchronization; event-related synchronization 


\section{Introduction}

Brain-computer interface (BCI) is an emerging technology that connects human brain to an output device, in order to communicate the cortical command signals to manipulate the actuator. These cortical signals are translated to device (e.g. computer) operatable commands [1]. The state-of-the art BCI is based on the idea of developing an artificial, muscle-free communication channel that acts as a natural communication channel between the brain and a machine $[2,3]$. Applications of BCI systems are widespread and vary from the fields of neuroscience, rehabilitation, cognitive infocommunications (CogInfoCom) [4] to entertainment, and defence [5]. Neurorehabilitation is the research area, which caters audiences with neurodegenerative disorders, spinal cord injury (SCI), amyotrophic lateral sclerosis (ALS) [6, 7], or lower-limb amputation [8]. The applications include neurorobotics, e.g. BCI-controlled wearable/assistive robots for mobility restoration. Such devices can be useful for direct communication in inter-cognitive CogInfoCom applications [2,9], and necessitates more research in this area.

In this study, the physiological signals used to detect natural cognitive capability of humans, are based on non-invasive modality, i.e. electroencephalography (EEG). We use this approach for its low cost and easy handling. When the human cognitive capability is combined with information and communication technologies (ICT), it results in an important aspect of CogInfoCom [10]. In order to connect high-level brain activity to infocommunication networks, BCI enables flow of rich information from the brain, and eventually heterogeneous cognitive entities into the ICT network [9, 10]. In this study, the source of information relevant to human cognitive states, include information on level of engagement during imagination of task and rest/idling, reflecting a decrease and increase in $m u$ wave $(8-12 \mathrm{~Hz})$ respectively [11].

Investigations on the possibility to use BCI system for post-stroke rehabilitation have been carried out in order to reinstate upper and lower-limb functions [12]. However, applications of existing BCI systems, for the control of various devices, such as a robotic exoskeleton, are not straightforward. One potential factor is the low dimensional control of these systems, i.e., they can only identify limited number of cognitive tasks as unique control commands. The most frequently used cognitive state motor imagery tasks, in a BCI system, are left hand vs. right hand, and foot kinesthesis motor imageries [13]. Successful control of cursor movement in two dimensions, on a computer screen, based on left vs. right hand motor imagery, was done by deploying the $m u(8-12 \mathrm{~Hz})$ and beta $(18-26 \mathrm{~Hz})$ rhythm, followed by several training sessions [14]. The same BCI cursor control strategy was extended to three-dimensions, where in addition to left-right hand imagery, foot motor imagery was incorporated, as well [15]. 
Successful quantification of left vs. right hand and foot motor imagery have been reported, including studies on the discrimination of different upper limbs [16], but no literature exists on the decoding of different movements within the same 'lower limb'. Investigations on independent lower-limb motor imagery tasks have been reported recently [2, 17-19], however, those studies did not cover the same limb tasks. This is because of the well-established fact about 'mesial wall' location of lower-limb representation area on the sensorimotor cortex. That precludes its exploitation during different imagery tasks. In addition to that, each joint representation within the same limb has a very close spatial representation to each other [20], which makes it difficult to discriminate each movement with electroencephalographic (EEG) signals.

In our research, we included foot and knee kinaesthetic imagery tasks within the same limb, as cognitive states. Each state was further divided into left vs. right imagery tasks, in order to increase the possibility for discriminating each task; thereby increasing the dimensionality of the BCI control signal. Recorded EEG signals, against each task, were quantified by observing the event related changes associated to the task in oscillatory $m u$ rhythm. The changes in oscillatory activity, with respect to an internally, or externally paced events, are time-locked, but not phase-locked, i.e. induced, known as event related desynchronization (ERD) or event related synchronization (ERS) [21, 22]. This study could be useful for the development of multi-dimensional control signals as a single cognitive entity in a BCI system for rehabilitation applications [9, 23]. Presented results are in accordance with an important aspect of CogInfoCom, i.e. the combination of the natural cognitive capability of human and ICT [24].

\section{Methods}

\subsection{Experimental Protocol}

This study was based on experiments performed on three healthy subjects with no history of neurological disorder, or any impairment. The age range was between 25-27 years, where all subjects participated on voluntary basis. None of the participants had any experience with BCI before. Ethics approval, for this research, was granted by the College Human Ethics Advisory Network (CHEAN) of RMIT University, Melbourne, Australia.

During the experiment every subject was directed to sit on a comfortable chair placed in front of a monitor screen (17') at a length of approximately $1.5 \mathrm{~m}$. The experimental protocol was based on the standard Graz protocol for synchronous BCI. Each trial began with a blank black screen that lasted for 30 seconds, in order to let the subject relax and get familiar with the environment. Following that, the 
trial began with the presentation of a green fixation cross on screen for 3 seconds (used as reference period for processing of epochs). One second long audio beep stimulus, right before the visual cue display, was incorporated in the first trial only, to alert the subject about the beginning of the experiment, see Figure 1 (left). Next, the visual cues of 2 seconds length were displayed followed by a 5 seconds long blank screen to perform the related task (imagery), making a total of 10 seconds for each trial. The visual cues in each trial reflected either the left or right movement. The foot and knee session was carried out separately. Our experimental paradigm consisted of alternate sessions, i.e. the first session for leftright foot kinaesthetic motor imagery (KMI), next session for left-right knee KMI, third for foot KMI and finally knee KMI. The cue set for each session is shown in figure 2. This was introduced to avoid a state of confusion for the subject with several tasks in a single session.

A standard one session protocol is composed of 40 trials, including 20 trials for each tasks, i.e. left or right KMI. The visual cues in each trial were displayed in a random order so that no adaptation could occur. Each trial was followed by a random pause interval of 1.5 to 3.5 seconds, in which the subjects were asked to rest. The experiment was divided into 4 sessions, i.e. foot, knee, foot and knee KMI respectively. Figure 1 (left) presents the schematic of experimental protocol reflecting the timing of cues, where each trial is 10 seconds long. For each session the respective visual cue set is given in figure 2, where (a) depicts left and right foot movements (dorsiflexion for 1 second) and (b) depicts left and right knee movements (extension for 1 second) respectively.

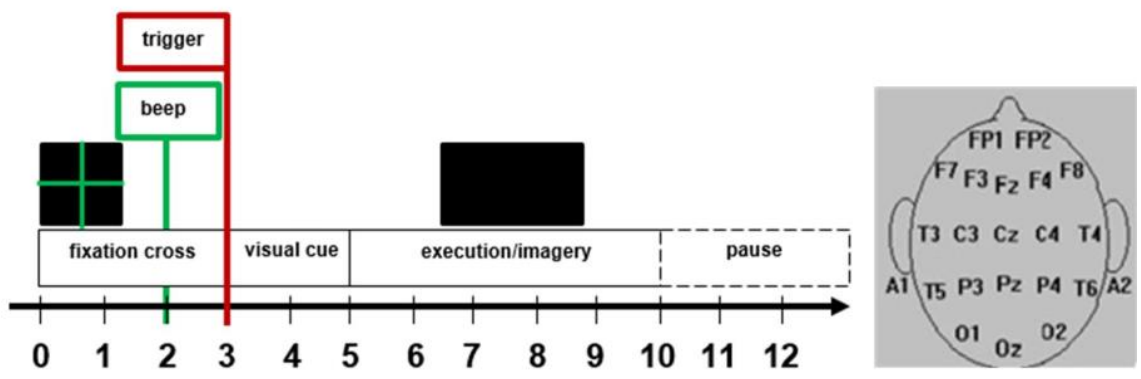

Figure 1

Experimental protocol timing in seconds (left) and 10-20 electrode channel locations (right) 

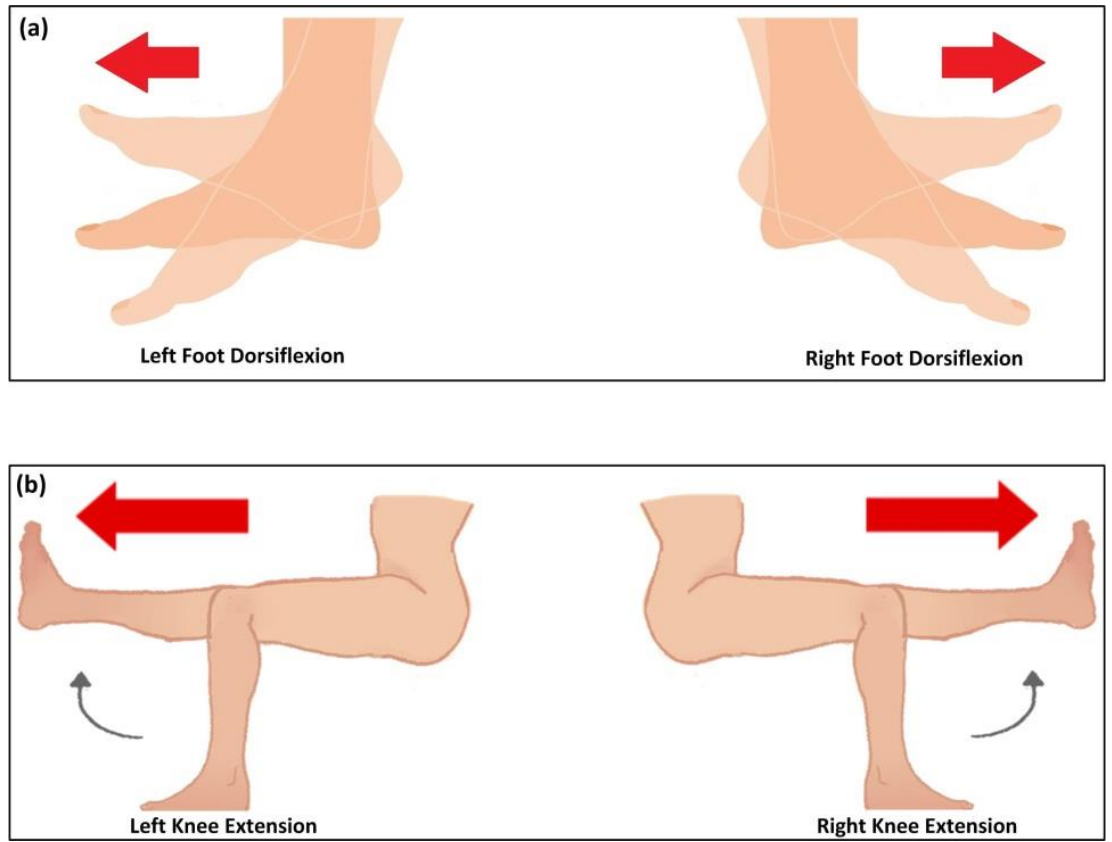

Figure 2

Visual cues in the experimental protocol, for (a) left - right foot dorsiflexion, and (b) left- right knee extension

\subsection{EEG Recording}

In order to record EEG activity, the EEG neurofeedback BrainMaster Discovery 24E amplifier (BrainMaster Technologies Inc., Bedford, USA) was utilised. The standard Graz synchronous BCI protocol was established using OpenViBE software (http://openvibe.inria.fr/downloads/) that also enabled the embedding of time stamps in each recorded trial. Overall experimental set up had the amplifier interfaced with the acquisition server of OpenViBE. To acquire brain signals from the motor cortex, the standard 10-20 Electro-cap was used [25]. The EEG system had 19 channels (10-20 sites), channel 20 (A2) was referenced to A1 (A2-A1) (Figure 1, right). Remaining channel including AUX1 and AUX2, provided for monitoring of other electrophysiological signals were not used. All channels were sampled using $256 \mathrm{~Hz}$ sampling frequency, with 24-bit resolution. The DC amplifier bandwidth was from $0.0 \mathrm{~Hz}$ to $100 \mathrm{~Hz}$, followed by EEG channel bandwidth from 0.43 to $80 \mathrm{~Hz}$.

The customized experimental protocol was designed using OpenViBE designer tool that comes along integrated feature boxes. The designer tool window is based on Lua script that was modified for generating customized scenario, Graz- 
Stimulator box was used to allow for the onset of different visual cue timings. Figure 3 reflects the connection established between the BrainMaster Discovery $24 \mathrm{E}$ and OpenViBE together synchronized. Each session was recorded in the standard EDF and GDF file formats using writer boxes of designer tool in OpenViBE.

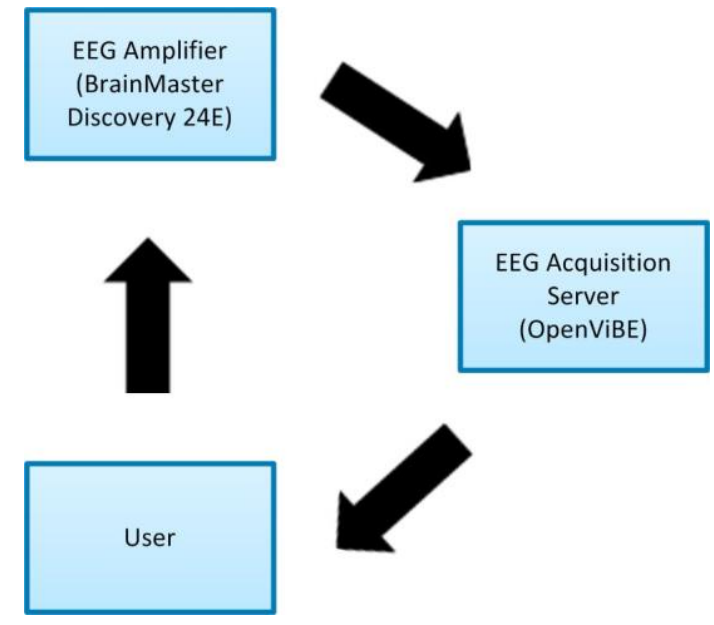

Figure 3

Established connection for real-time EEG data acquisition and incorporation of event time-stamps in the data stream

\subsection{Signal Processing}

In order to process and visualize the acquired data offline, the statistical EEGLAB package was used. During offline processing, the EEG data was converted to reference-free form by using the common average reference method. The data was pre-processed using FIR bandpass filter between $8-12 \mathrm{~Hz}$, which was the required frequency bandwidth range for $m u$ rhythm. Next, each epoch, i.e. trial of 10 seconds length was extracted, which included 3 seconds period prior to cue onset, to be used as reference period during analysis.

The epoched data was then filtered using spatial filter, i.e. the independent component analysis (ICA) for artifacts removal.

For each subject, spectral plots were generated that reflected the 2-class statistics, where each class was related to each task. Following this, the average time course ERD and ERS for $m u$ rhythm $(8-12 \mathrm{~Hz})$ were plotted, where only statistically significant ERD/ERS were displayed. This was done using validation method to ensure statistically significant data, i.e. to allow assigning measures of accuracy (confidence interval) to sample estimates. We used the bootstrap statistical 
significance method, with confidence interval of $95 \%$. In this way the significant ERD-ERS features were selected. The central electrode areas $\mathrm{C} 3, \mathrm{Cz}$, and $\mathrm{C} 4$ linked to sensorimotor cortex were used to analyse $m u$ band with the most significant bandpower decrease, or increase, during each task.

The standard procedure for calculation of ERD/ERS patterns was adopted from [26]. After bandpass filtering of each trial, the samples were squared and subsequently averaged over trials and over sample points [27]. This directed to the resulting proportional power decrease (ERD), or power increase (ERS) compared to the reference interval, which was selected as the period of 3 seconds before the trigger onset of visual cues. In order to overcome masking of induced activities caused by the evoked potentials, the mean of the bandpass filtered data was subtracted from the data for each sample [28].

The ERD/ERS was calculated from EEGLAB [29, 30] integrated function eventrelated spectral perturbations (ERSP) based on wavelet decomposition. ERSP detects the event-related shifts in the power spectrum. It measures the mean eventrelated changes in the power spectrum at one data channel averaged over trials. $P_{j}$ is the power or intertrial variance of the $j^{\text {th }}$ sample and $R$ is the average power in the reference interval $\left[r_{0}, r_{0}+k\right]$. To convert ERSP to ERDS, equations 1 and 2 were used; ERSP was normalized to the reference interval [29]:

$$
\begin{aligned}
& E R S P_{j}=10 \log \left(\frac{P_{j}}{R}\right) \\
& E R D S_{j}=\left(10^{\frac{E R S P_{j}}{10}}-1\right) \times 100
\end{aligned}
$$

\section{Results}

The results obtained from all three subjects, s1, s2 and s3 are presented in this section.

\subsection{ERD/ERS Quantification}

In order to quantify the significant cognitive bandpower changes of $m u$ rhythm, each combination of lower-limb tasks was pre-processed and spatial filter was applied on the filtered data. Resulting signals were evaluated for each central electrode position directing towards the sensorimotor cortex, and the potential area where $m u$ rhythm elicits. Table 1 shows the illustration of quantification approach. 
Table 1

Unsupervised feature extraction-based approach

\begin{tabular}{|c|c|c|c|c|c|}
\hline Tasks & $\begin{array}{c}\text { Pre- } \\
\text { processing }\end{array}$ & $\begin{array}{l}\text { Spatial } \\
\text { filter }\end{array}$ & $\begin{array}{l}\text { Scalp } \\
\text { location }\end{array}$ & $\begin{array}{c}\text { Time-frequency } \\
\text { feature } \\
\text { extraction }\end{array}$ & \\
\hline LF vs. LK & \multirow{3}{*}{$\begin{array}{l}\text { Bandpass } \\
\text { filtering, } \\
\text { Epoching }\end{array}$} & \multirow{3}{*}{ ICA } & C3 & \multirow{3}{*}{$\begin{array}{l}\text { Wavelet (short- } \\
\text { time DFT) } \\
\text { transform }\end{array}$} & \\
\hline RF vs. RK & & & $\mathrm{C} 4$ & & \multirow[t]{2}{*}{ Avg } \\
\hline $\begin{array}{c}\text { LF-LK vs. } \\
\text { RF-RK }\end{array}$ & & & $\mathrm{Cz}$ & & \\
\hline
\end{tabular}

\subsubsection{Spectral Topographical Plots}

The cognitive state output, in the form of percentage power ERD and ERS spectral maps, for all participants, against the foot and knee tasks for each session respectively, were plotted between $8-12 \mathrm{~Hz}$ frequency of $m u$ band. Each session comprised of left-right tasks of foot followed by knee, i.e. different movements within the same limb. Figure 4 represents the topographical scalp plots of each subject during left-right foot and left-right knee imagery respectively, for 8 to 12 $\mathrm{Hz}$.

For s1, it was observed that during left foot, and left knee, imagery tasks, the foot as well as hand area $m u$ rhythm $(m u$ ERD) was enhanced in both cases. However, with left foot imagery the ERD was localized towards left hemisphere, C3, whereas the left knee imagery showed broad-banded ERD towards central area $\mathrm{Cz}$ and edged towards parietal region. The right foot and knee tasks, in the same limb somehow revealed similar output. However, with right foot imagery prominent $m u$ ERD overlying the primary hand area was observed, where ERD was dominantly visible at electrode position $\mathrm{C} 3$ in addition to $\mathrm{Cz}$. This pointed towards the possibility of contralateral spectral power dominance during right foot task. On the other hand, the right knee imagery depicted an enhancement in the $m u$ ERD foot area representation edged towards parietal region.

The left foot imagery with s2 enhanced the ERD patterns at central electrode positions predominantly $\mathrm{C} 3$, similar to $\mathrm{s} 1$, as well as the premotor areas. This was not the case with left knee imagery task, which did not exhibit enhancement in power concentration. Following this, during the right foot imagery an overall increase in mu ERD power concentration was observed over the primary, supplementary and pre-motor areas with contralateral dominance. Interestingly a small increase in $m u$ ERS spectral power was visible during the right knee imagery task, which was strictly localized towards the central and parietal regions. This directed towards no prominent ERD.

The resulting plots of s3, during left foot task, elicited power concentration in ERD focused towards the hand and foot area. However, the left knee imagery depicted a very clear focal enhancement in $m и$ ERD foot area representation. 
During the right foot task, a higher power concentration in $m u$ ERD overlying the central cortical regions with a shift towards parietal area was visible. Similarly, the right knee task, elicited increased power ERD strictly in cortical foot area, at central region of the cortex. No contralateral power distribution was visible with subjects 2 and 3 .

\subsubsection{ERD/ERS Average Time Course in $m u$ Rhythm}

The resulting cognitive states, in form of ERD/ERS time course for $m u$ rhythm with frequency range of $8-12 \mathrm{~Hz}$ at electrode positions $\mathrm{C} 3, \mathrm{C} 4$, and $\mathrm{Cz}$ are shown in Figure 5. The results elicited by $\mathrm{s} 1$ are presented.

In order to compute the specified time and frequency resolution, i.e. averaging over sample points, the EEGLAB integrated sinusoidal wavelet transform (shorttime discrete fourier transform (DFT)) was used. A $t$ percentile bootstrap statistic (percentile taken from baseline distribution, with a significance level of $\alpha=0.05$, was applied to get significant ERD and ERS values [29]. The basic aim of bootstrap technique is to replace the unknown population distribution with a known empirical distribution and based on the empirical distribution estimator, determine the confidence interval, in this case $95 \%$ confidence [21].

Different movements within the same lower-limb elicit various percentage power ERD and ERS. Figure 5 reflects each combination of tasks for different joint positions, within the same lower-limb. The selection of central electrode position, for plotting each combination of tasks, within the same limb, was based on the probability to observe any contralateral dominance in the power concentration ERD. Therefore, C3 was selected for observing right imagery task characteristic ERD within the same limb. C4 was selected to detect left task characteristic ERD, $\mathrm{Cz}$ was chosen to observe left and right task ERD characteristics and their impact on the midline of the central lobe for each participant. The task combinations within the same lower-limb are given in Table 2.

Table 2

Task combinations within the same lower-limb

\begin{tabular}{lll}
\hline Electrode position & Mental task & Bandpower features \\
\hline $\mathrm{C} 3$ & Imagery right foot vs. right knee & ERDS average \\
\hline $\mathrm{C} 4$ & Imagery left foot vs. left knee & ERDS average \\
\hline $\mathrm{Cz}$ & $\begin{array}{l}\text { Imagery right foot-knee vs. } \\
\text { left foot-knee }\end{array}$ & ERDS grand average \\
\hline
\end{tabular}

At C3 during right foot and knee imageries, ERD time course was obtained by taking average of power changes in $m u$ rhythm across all trials with each subject. At the end of visual cue (shown by green window in Figure 5), the $т и$ power attenuates for approximately 0.6 seconds, after onset of cue. Evident ERS was visible at approximately 3 seconds, which is referred to the period of task 
performance. Since each of the foot dorsiflexion and knee extension task, were 1 second in length, the appearance of an ERS at 3 seconds correlates to the completion of task by the subject.

The left foot and knee imagery movements at electrode position $\mathrm{C} 4$ did not depict a very prominent ERD. However, at the beginning of cue onset at approximately 0.3 seconds a desynchronization of the foot area is visible followed by another dip at approximately 4 seconds (imagery interval). ERS was visible between 4 and 5 seconds towards the termination of the task performance interval.

Finally, at electrode position $\mathrm{Cz}$, most dominant percentage power decrease, ERD was visible throughout the beginning of visual cue onset window followed by the task performance interval. These results are in accordance with the established results from the spectral power distribution maps. The presence of large centrally localized ERD patterns validates the notion of enhanced foot $m u$ area representation elicited by $\mathrm{Cz}$ upon foot and knee imagery related tasks.

Clear results at $\mathrm{Cz}$ were due to the grand average taken for all four trials and sessions for each participant, which was not the case with $\mathrm{C} 3$ and $\mathrm{C} 4$, where the average of each trial and session for only two tasks was taken.

The grand-average amplitude of $m u$ ERD for all subjects based on common average reference derivation at central electrode positions is shown in figure 6 . The error bars represent the standard deviation. As depicted earlier from results, there was no significant inter-task difference within the same limb, observed at electrode positions $\mathrm{C} 3, \mathrm{Cz}$ and $\mathrm{C} 4(P<0.05, t$-test). However, it is important to mention here that the bar graphs were only plotted for $т и$ ERD and not ERS, to infer knowledge about its behavior output. Taking beta ERD/ERS features into account could add to the overall information during lower-limb tasks within the same limb. 

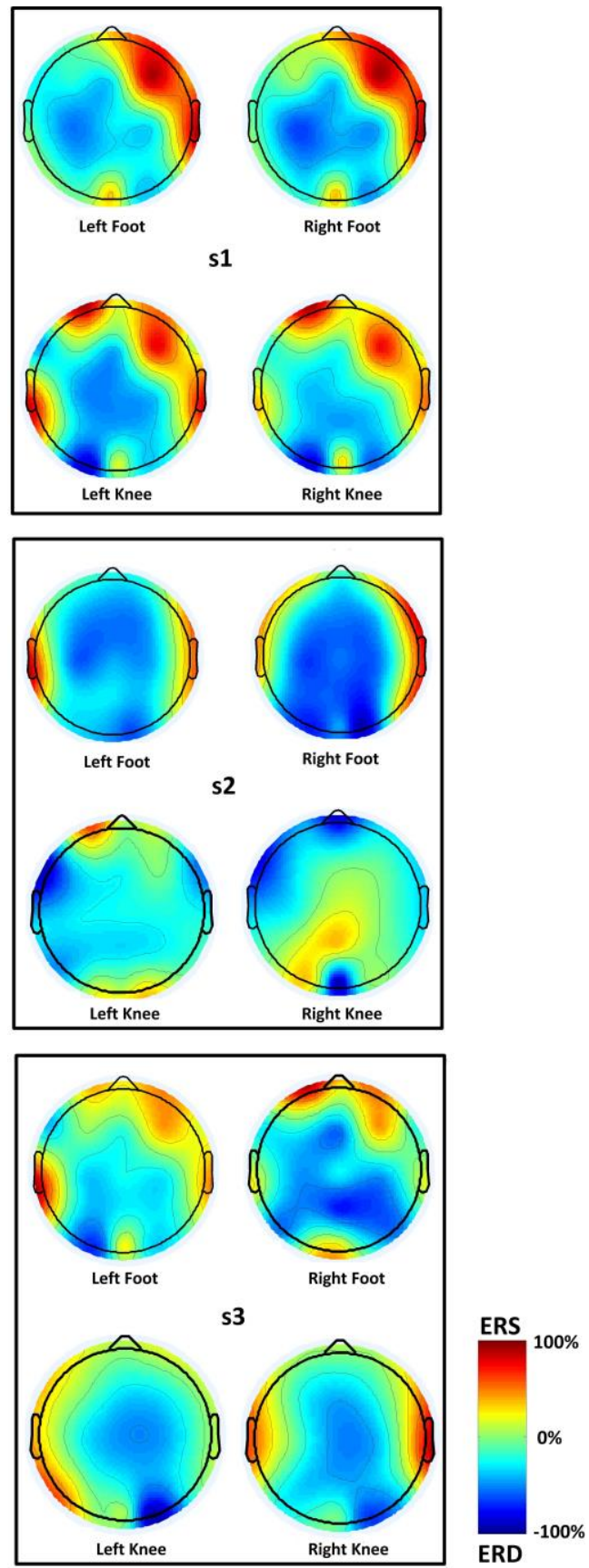

Figure 4

Topographical scalp maps of each subject during left-right foot and left-right knee imagery respectively between frequencies of $8-12 \mathrm{~Hz}$ 

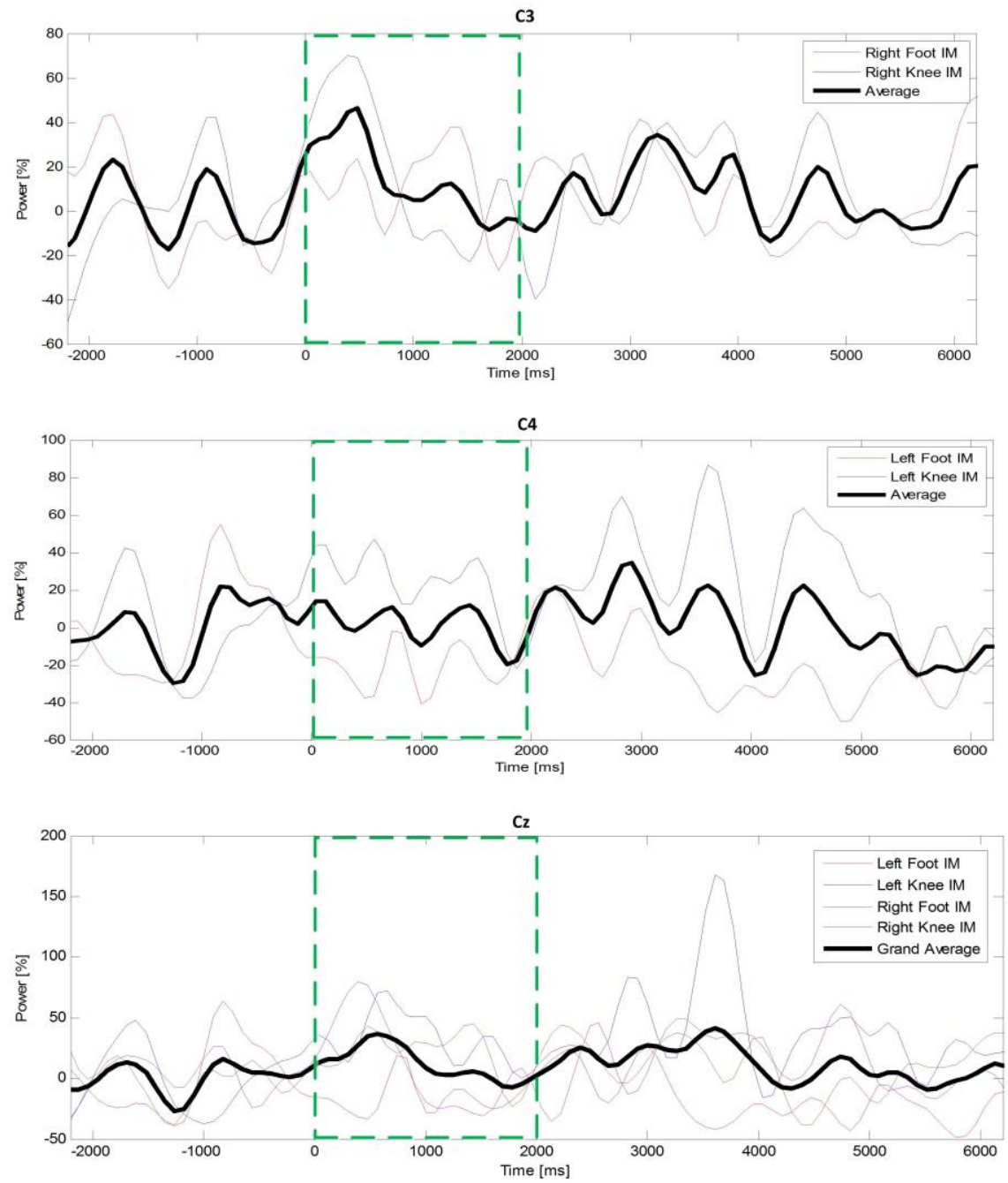

Figure 5

ERD and ERS time course for $m u$ rhythm $(8-12 \mathrm{~Hz})$ of subject 3 at electrode position $\mathrm{C} 3$ for right foot and right knee imagery alongside their average, $\mathrm{C} 4$ for left foot and left knee imagery alongside their average, and $\mathrm{Cz}$ for left and right foot and knee imagery respectively alongside their average. The green window indicates visual cue presentation from 0 and 2 seconds 


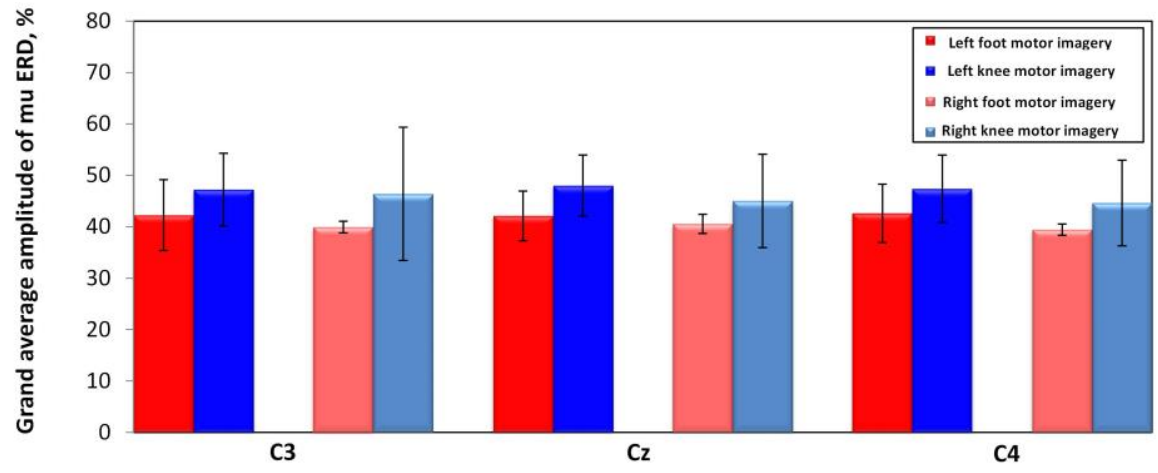

Figure 6

Average amplitude of $m u$ ERD from all subjects based on common average reference derivation at central electrode positions. The red and blue bars indicate left foot and left knee motor imageries, respectively, and pale red and pale blue bars indicate right foot and right knee motor imageries, respectively. Error bars represent standard deviations

\section{Discussion}

We analysed the discrimination of cognitive states, as a result of imaginary leftright foot and knee motor tasks within the same limb. It was observed that an increase in power concentration of $m u$ ERD overlying hand and foot area occured with majority of the subjects. Although the hand area in this study was not needed to perform a task, we therefore, consider it to be in an idling state. Generally, no explicit contralateral dominance was visible, except for s1 and s2, who both showed contralateral dominance during right foot imagery task at $\mathrm{C} 3$. As foot, the knee area representation is also situated in the mesial wall, which makes it difficult to elicit clear ERD patterns upon knee imagery tasks. However, with left and right knee discrimination tasks, in all subjects, centrally localized ERD patterns were mainly observed throughout. The focal $m u$ rhythm was visible in cortical foot representation area with small activation of hand area with s1 only during left knee imagery.

For neurorobotics and human ICT applications, this can lead to the inference that kinaesthetic knee imagery blocks or desynchronizes foot area $m u$ rhythm, at central electrode positions and shifts over supplementary, pre-motor areas and in some cases towards parietal region. Results suggest that the cortical knee representation area is situated near the foot sensorimotor areas. The other task in same lower-limb, i.e., foot motor imagery, not only activated hand and foot area ти ERD but also elicited contralateral dominance during right foot kinaesthetic imagery. The knee kinaesthetic imagery on the other hand does not provide enough evidence of contralateral dominance of the cognitive states upon left vs. 
right imagery tasks. This was also validated by the average $m u$ ERD bar graph, that reflected difference during left-right foot tasks but no significant difference during the knee tasks. More investigations in this area could be very useful for CogInfoCom based systems to highlight the activeness of specific brain regions indicating human level engagement in biofeedback-driven frameworks.

\section{Conclusions and Future Work}

This research broadened new horizons towards investigation of cognitive states as event-related changes in oscillatory activity of $m u$ during foot and knee motor imageries within the same lower-limb. The results provide useful information on human level of engagement during imagination of task and rest, as reflected by $m u$ rhythm activity. Despite a small lower-limb sensorimotor area representation in the homunculus, the foot and knee movement imagery elicited ERD patterns. Based on the spectral power plots, an increase in the mid-central ERD was observed overall with all the subjects. The kinaesthetic knee imagery triggered $m u$ ERD, mainly in the cortical foot area representation, with small shift towards parietal lobe. No contralateral dominance of cortical areas was present in the case of left-right knee imagery tasks, unlike with foot tasks. Obtained results suggest that intra-subject cognitive-state variability exists during the reactivity of $m u$ components. This makes it difficult to draw a clear difference between different lower-limb tasks within the same limb. However, clear results with one subject; indicate the possibility of discriminating different movements within the same lower-limb. Suggested protocol could be exploitable to increase the dimensionality of control signals, as a cognitive entity, in a BCI system. Involvement of more participants and classification of feature vector is the future aim of this investigation, to develop a multi-dimensional CogInfoCom tool for BCI controlled devices.

\section{Acknowledgement}

This work is supported by RMIT University through international post graduate research scholarship (IPRS).

\section{References}

[1] Wolpaw, J. R., et al., Brain-computer interfaces for communication and control. Clinical neurophysiology, 2002. 113(6): pp. 767-791

[2] Tariq, M., et al. Mu-beta rhythm ERD/ERS quantification for foot motor execution and imagery tasks in BCI applications. in Cognitive Infocommunications (CogInfoCom), $2017 \quad 8^{\text {th }}$ IEEE International Conference on. 2017, IEEE

[3] Henshaw, J., W. Liu, and D. M. Romano. Improving SSVEP-BCI performance using pre-trial normalization methods. in Cognitive 
Infocommunications (CogInfoCom), $2017 \quad 8^{\text {th }}$ IEEE International Conference on. 2017, IEEE

[4] Garcia, A. P., I. Schjølberg, and S. Gale. EEG control of an industrial robot manipulator. in Cognitive Infocommunications (CogInfoCom), 2013 IEEE $4^{\text {th }}$ International Conference on. 2013, IEEE

[5] Katona, J., et al. Speed control of Festo Robotino mobile robot using NeuroSky MindWave EEG headset based brain-computer interface. in Cognitive Infocommunications (CogInfoCom), $20167^{\text {th }}$ IEEE International Conference on. 2016, IEEE

[6] Vaughan, T. M., J. R. Wolpaw, and E. Donchin, EEG-based communication: Prospects and problems. IEEE transactions on rehabilitation engineering, 1996, 4(4): pp. 425-430

[7] Millán, J. d. R., et al., Combining brain-computer interfaces and assistive technologies: state-of-the-art and challenges. Frontiers in neuroscience, 2010, 4: p. 161

[8] Tariq, M., Z. Koreshi, and P. Trivailo. Optimal Control of an Active Prosthetic Ankle. in Proceedings of the $3^{\text {rd }}$ International Conference on Mechatronics and Robotics Engineering. 201, ACM

[9] Baranyi, P. and A. Csapo, Definition and synergies of cognitive infocommunications. Acta Polytechnica Hungarica, 2012, 9(1): pp. 67-83

[10] Baranyi, P., A. Csapo, and G. Sallai, Cognitive Infocommunications (CogInfoCom) 2015: Springer

[11] He, B., et al., Noninvasive brain-computer interfaces based on sensorimotor rhythms. Proceedings of the IEEE, 2015, 103(6): pp. 907-925

[12] Ang, K. K. and C. Guan, Brain-computer interface in stroke rehabilitation. Journal of Computing Science and Engineering, 2013, 7(2): pp. 139-146

[13] Pfurtscheller, G. and C. Neuper, Motor imagery and direct brain-computer communication. Proceedings of the IEEE, 2001, 89(7): pp. 1123-1134

[14] Wolpaw, J. R. and D. J. McFarland, Control of a two-dimensional movement signal by a noninvasive brain-computer interface in humans. Proceedings of the National Academy of Sciences of the United States of America, 2004, 101(51): pp. 17849-17854

[15] Royer, A. S., et al., EEG control of a virtual helicopter in 3-dimensional space using intelligent control strategies. IEEE Transactions on neural systems and rehabilitation engineering, 2010, 18(6): pp. 581-589

[16] Yong, X. and C. Menon, EEG classification of different imaginary movements within the same limb. PloS one, 2015, 10(4): p. e0121896

[17] Tariq, M., P. M. Trivailo, and M. Simic. Detection of knee motor imagery by $\mathrm{Mu}$ ERD/ERS quantification for BCI based neurorehabilitation applications. in Control Conference (ASCC), $201711^{\text {th }}$ Asian. 2017, IEEE 
[18] Tariq, M., P. M. Trivailo, and M. Simic, Event-related changes detection in sensorimotor rhythm. International Robotics \& Automation Journal, 2018, 4(2): pp. 119-120

[19] Pfurtscheller, G. and T. Solis-Escalante, Could the beta rebound in the EEG be suitable to realize a "brain switch"? Clinical Neurophysiology, 2009, 120(1): pp. 24-29

[20] Plow, E. B., et al., Within-limb somatotopy in primary motor cortexrevealed using fMRI. Cortex, 2010, 46(3): pp. 310-321

[21] Graimann, B., et al., Visualization of significant ERD/ERS patterns in multichannel EEG and ECoG data. Clinical Neurophysiology, 2002, 113(1): pp. 43-47

[22] Pfurtscheller, G. and F. L. Da Silva, Event-related EEG/MEG synchronization and desynchronization: basic principles. Clinical neurophysiology, 1999, 110(11): pp. 1842-1857

[23] Izsó, L. The significance of cognitive infocommunications in developing assistive technologies for people with non-standard cognitive characteristics: CogInfoCom for people with non-standard cognitive characteristics. in Cognitive Infocommunications (CogInfoCom), $20156^{\text {th }}$ IEEE International Conference on. 2015, IEEE

[24] Baranyi, P., A. Csapo, and P. Varlaki. An overview of research trends in CogInfoCom. in Intelligent Engineering Systems (INES), $201418^{\text {th }}$ International Conference on. 2014, IEEE

[25] Klem, G. H., et al., The ten-twenty electrode system of the International Federation. Electroencephalogr Clin Neurophysiol, 1999, 52(3): pp. 3-6

[26] Kalcher, J. and G. Pfurtscheller, Discrimination between phase-locked and non-phase-locked event-related EEG activity. Electroencephalography and clinical neurophysiology, 1995, 94(5): pp. 381-384

[27] Knösche, T. R. and M. C. Bastiaansen, On the time resolution of eventrelated desynchronization: a simulation study. Clinical Neurophysiology, 2002, 113(5): pp. 754-763

[28] Graimann, B. and G. Pfurtscheller, Quantification and visualization of event-related changes in oscillatory brain activity in the time-frequency domain. Progress in brain research, 2006, 159: pp. 79-97

[29] Delorme, A. and S. Makeig, EEGLAB: an open source toolbox for analysis of single-trial EEG dynamics including independent component analysis. Journal of neuroscience methods, 2004, 134(1): pp. 9-21

[30] Delorme, A., et al., EEGLAB, SIFT, NFT, BCILAB, and ERICA: new tools for advanced EEG processing. Computational intelligence and neuroscience, 2011, 2011: p. 10 